\title{
Nationwide trends in glucose-lowering drug use, Denmark, |999-2014
}

\author{
Diana Hedevang \\ Christensen' \\ Jørgen Rungby ${ }^{2,3}$ \\ Reimar Wernich Thomsen' \\ 'Department of Clinical \\ Epidemiology, Aarhus University \\ Hospital, Denmark; ${ }^{2}$ Department of \\ Pharmacology, University of Aarhus, \\ Aarhus, Denmark; ${ }^{3}$ Department of \\ Endocrinology, Gentofte University \\ Hospital, Copenhagen, Denmark
}

Correspondence: Diana Hedevang

Christensen

Department of Clinical Epidemiology,

Aarhus University Hospital, Olof Palmes

Allé 43-45, DK-8200 Aarhus Nord,

Denmark

Tel +4587168248

Fax +4587167215

Email dhcr@clin.au.dk
This article was published in the following Dove Press journal:

Clinical Epidemiology

14 October 2016

Number of times this article has been viewed

Purpose: The objective of this study was to examine nationwide population-based time trends in the utilization of all glucose-lowering drugs in Denmark from 1999 to 2014.

Methods: Based on nationwide data from the Register of Medicinal Products Statistics, we retrieved sales statistics on glucose-lowering drugs and reported the total number of users and the prevalence of users per 1,000 inhabitants in 1-year intervals for all glucose-lowering drug classes. Results: The annual prevalence of glucose-lowering drug users increased more than twofold from 19 per 1,000 inhabitants in $1999(n=98,362)$ to 41 per 1,000 in $2014(n=233,230)$. Metformin use increased more than sevenfold during the period and was used by 30 of 1,000 inhabitants in 2014, while the prevalence of insulin use increased 1.8-fold to 13 per 1,000 in 2014. After peaking in 2007, use of sulfonylurea halved to 6 per 1,000 in 2014. Newer drug classes including the glucagon-like peptide 1 receptor agonists, the dipeptidylpeptidase- 4 inhibitors, and the sodium-glucose cotransporter 2 inhibitors had reached a considerable position by 2014 , with 4 per $1,000,6$ per 1,000 , and 0.8 per 1,000 inhabitants, respectively; however, the use of glucagon-like peptide 1 receptor agonists and sodium-glucose cotransporter 2 inhibitors in elderly people remained low. Thiazolidinediones decreased to virtually no use $(0.03$ per 1,000) in 2014.

Conclusion: The use of glucose-lowering drugs has doubled during 1999-2014. The pattern of glucose-lowering drug use has changed substantially reflecting the recommendations of metformin as first-line treatment. The newer glucose-lowering drug classes have been well received.

Keywords: drug utilization, registries, diabetes mellitus, antidiabetic drugs

\section{Introduction}

Over the past 10-15 years, treatment guidelines have changed emphasizing early initiation of pharmacotherapy in type 2 diabetes mellitus (type 2DM). ${ }^{1-3}$ Metformin is the preferred first-line therapy, ${ }^{1-3}$ and early and individualized intensification is recommended if the hemoglobulin A1c goal is not reached. ${ }^{1-3}$ Concurrently, the therapeutic armamentarium has expanded, with many novel glucose-lowering treatment options, eg, the glucagon-like peptide 1 (GLP-1) receptor agonists (2006), the dipeptidylpeptidase-4 (DPP-4) inhibitors (2007), the sodium-glucose cotransporter 2 (SGLT-2) inhibitors (2012), and several new types of insulin such as glargine (2000), detemir (2004), and degludec (2013) emerging. Considering these changes, unselected population-based prescription data are important to monitor shifting trends in glucose-lowering drug use in large-scale real-world populations. With an estimated 320,000 people currently living with diabetes in Denmark ( $\approx 6 \%$ of the population; $>90 \%$ with type $2 \mathrm{DM}$ ), 
increasing from 140,000 persons with diabetes in $1999^{4}$ - and with comprehensive registration of health data - Denmark provides a solid platform to conduct such studies. Here, we report nationwide time trends in the utilization of glucoselowering drugs in Denmark from 1999 to 2014.

\section{Methods}

\section{Setting and population data sources}

The Danish National Health Service provides all Danish residents with equal tax-supported health care including partial reimbursement for prescribed medications, ${ }^{5}$ such as glucoselowering drugs. The unique personal identification number (the so-called civil personal registration [CPR] number) assigned to each Danish citizen at birth or upon immigration allows accurate individual-level linkage across Danish social service and health databases. ${ }^{5}$

We used the web facility Medstat to obtain data on the sale of glucose-lowering drugs in Denmark from 1999 onward. ${ }^{6}$ This publicly accessible webpage from the Danish Health Data Authority provides aggregate statistics on sale of pharmaceuticals in Denmark, based on individual-level data. ${ }^{6}$ The main population-based data source for these statistics is the Register of Medicinal Products Statistics (RMPS), which has collected records on sales of all medicinal products nationwide, including individual-level data on all outpatient dispensations, since $1995 .{ }^{6,7}$ Danish community pharmacies are essentially monopolized for prescription drug sales and equipped with electronic accounting systems, used to secure reimbursement, which electronically transmit data to the National Health Service and RMPS. ${ }^{7,8}$ Data include the patient's unique CPR number (encoding sex and age), date of sale, type of drug, strength and package size, and defined daily dose (DDD). ${ }^{7-9}$ Because each dispensation is identified by the CPR number, it is possible to study drug utilization by patient age, sex, and place of residence via linkage to the Civil Registration System. Reporting to RMPS is obligatory, and since 1999, Medstat statistics have been complete nationwide. In our study, a person was included as user of a specific glucose-lowering drug when having bought that drug at least once in the year concerned.

\section{Statistical analyses}

We obtained primary health care sector sales statistics from January 1, 1999, to December 31, 2014, for the entire Danish population, $\sim 5.6$ million inhabitants by 2014 . We reported the absolute number of users of glucose-lowering drug classes as well as the prevalence proportion per 1,000 inhabitants for each group of glucose-lowering drugs in 1-year intervals and graphically illustrated the trends overall and stratified according to sex and age ( $<20$ years, 20-39 years, 40-64 years, $65-79$ years, and $\geq 80$ years). Age was defined as the age at first redeemed prescription each year. We calculated the proportion of total glucose-lowering drug users who used each specific drug class in the first and last study year.

\section{Research ethics and informed consent}

As this study was based solely on register data and did not involve any contact with patients, no approval or informed consent was required from the Danish Scientific Ethical Committee

\section{Results}

The annual prevalence of glucose-lowering drug users increased more than twofold from 19 per 1,000 inhabitants $(n=98,362)$ in 1999 to 41 per $1,000(n=233,230)$ in 2014. Metformin was by far most frequently used, prescribed in $72 \%$ of all persons using glucose-lowering drugs in 2014, followed by insulin prescribed in $33 \%$ of all persons (Figure 1 and Table 1). The annual prevalence of metformin users increased more than sevenfold from 4 per $1,000(n=22,738)$ in 1999 to 30 per $1,000(n=167,316)$ in 2014 . The increase in metformin users leveled off during the last 3 years. Sulfonylureas were the third most commonly used glucose-lowering drugs in $2014(\mathrm{n}=35,435$ users, 15\% of all users) after a substantial decrease since their peak in 2007 . In contrast, prescribing of DPP-4 inhibitors increased steadily since their introduction in 2007; in 2014, 15,680 (3 per 1,000) used a combination pill of metformin and a DPP-4 inhibitor and 17,444 (3 per 1,000) redeemed a prescription of a DPP-4 inhibitor noncombination pill. Prescribing of GLP-1 receptor agonists increased rapidly immediately after introduction and stabilized during the last 2 years, with 4 per $1,000(n=19,947)$ users in $2014(9 \%$ of all users). SGLT-2 inhibitor use reached 0.8 per 1,000 (123 using a combination pill of metformin and SGLT-2 inhibitor and 4,398 using a noncombination pill) already within the third year after introduction to the Danish market. The 1-year prevalence of thiazolidinedione users peaked in 2007 with 3,744 persons $(0.7$ per 1,000$)$ using a combination pill with metformin and 1,576 users of noncombination pills dropping to only 154 users $(0.03$ per 1,000) in 2014. Sales of alpha-glucosidase inhibitors and meglitinides remained low over time.

Despite a steadily increasing absolute number of insulin users, the proportion of all glucose-lowering drug users who used insulin declined from $41 \%$ in 1999 to $33 \%$ in 2014. The use of insulin subtypes also changed substantially (Figure 1 

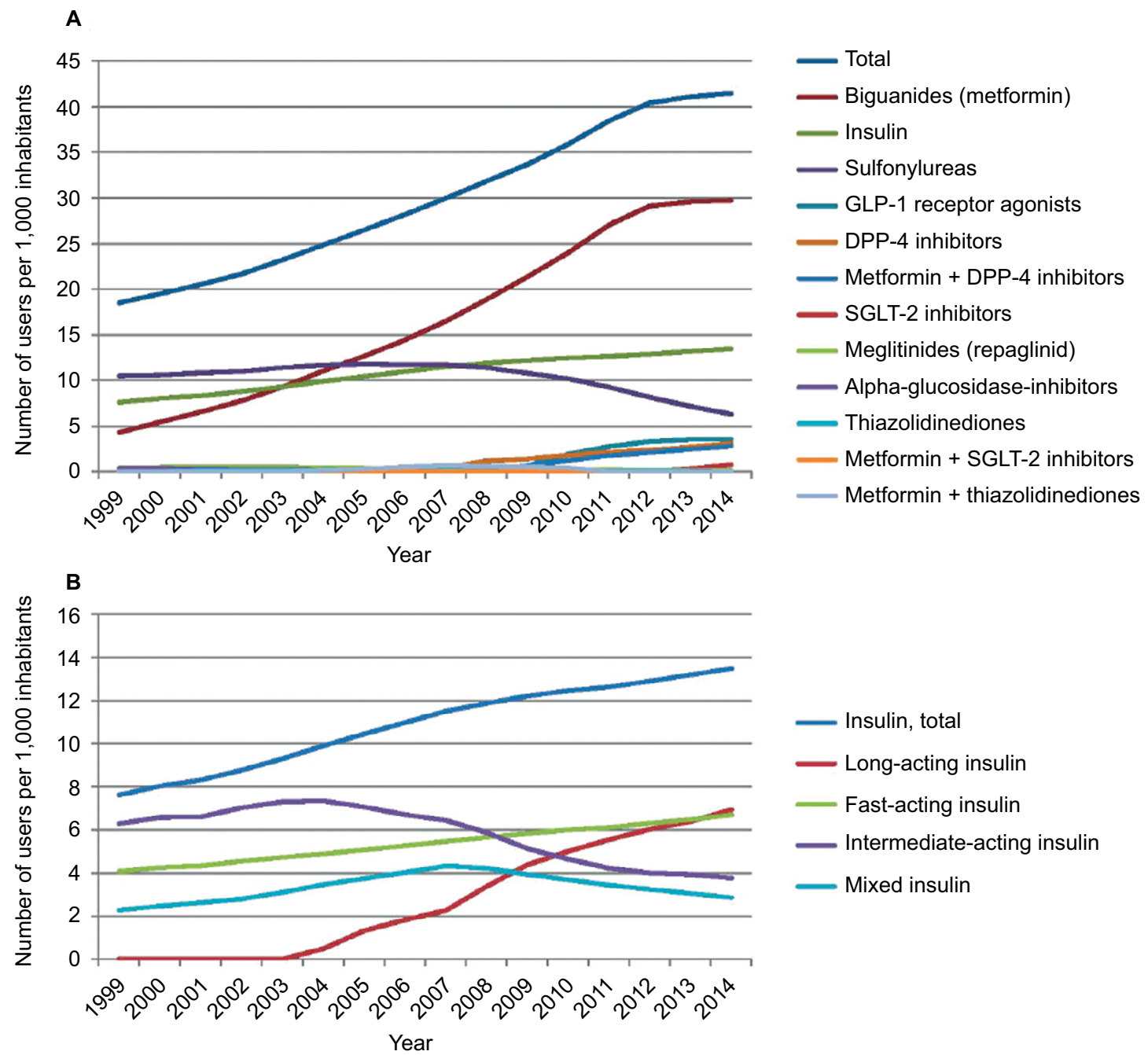

— Insulin, total

_ Long-acting insulin

— Fast-acting insulin

_ Intermediate-acting insulin

— Mixed insulin

Figure I Annual prevalence of all glucose-lowering drug-users (A) and insulin users (B) in Denmark per I,000 inhabitants, 1999-2014.

Notes: The number of persons prescribed different therapies in a given year is higher than the total number of glucose-lowering drug users that year due to possibility of polypharmacy and drug shifters. Mixed insulin cover insulin preparations consisting of intermediate- or long-acting insulin in combination with fast-acting insulin.

Abbreviations: GLP-I, glucagon-like peptide I; DPP-4, dipeptidylpeptidase 4; SGLT-2, sodium-glucose cotransporter 2.

and Table 2). The prevalence of long-acting insulin users increased from 2004 onward reaching 7 per $1,000(n=39,019$, $51 \%$ of all insulin users) in 2014, whereas the prevalence of intermediate-acting and mixed formula insulin users declined. The number of fast-acting insulin users increased from 4 per 1,000 ( $\mathrm{n}=22,670,54 \%$ of all insulin users) in 1999 to 7 per $1,000(n=37,718,50 \%$ of all insulin users $)$ in 2014 .

\section{Sex- and age-stratified results}

Stratified results showed a very similar development in men and women (Tables S1-S4 and Figures S1 and S2). Prescribing of metformin accelerated from 2006 onward, in particular in elderly people (Tables S5-S9 and Figures S3-S7). Prescribing of the new glucose-lowering drug classes increased in all age groups older than 20 years. However, GLP-1 receptor agonist use increased most among 40-64 years old and $65-79$ years old persons reaching 6 per 1,000 (12\% of all users) and 9 per 1,000 ( $8 \%$ of all users), respectively, by 2014 , versus 2 per 1,000 ( $1.5 \%$ of all users) in those aged 80+ (Tables S7-S9 and Figures S5-S7). A similar age-dependent use was observed for the SGLT-2 inhibitors, whereas a comparable proportion of glucose-lowering drug users used DPP-4 inhibitors among those aged 40-64 years, 65-79 years, and 80+ years in 2014 (Tables S7-S9 and Figures S5-S7).

A stable prevalence of insulin users was observed for those aged 20-39 years (5.5 per 1,000 in 1999 and 6 per 1,000 in 2014), whereas the number increased in the remaining age groups. Solely, the number of fast-acting insulin users steadily increased in the youngest group, whereas prescribing of fast-acting and in particular long-acting insulin increased in the other age groups. Use of mixed formula insulin 


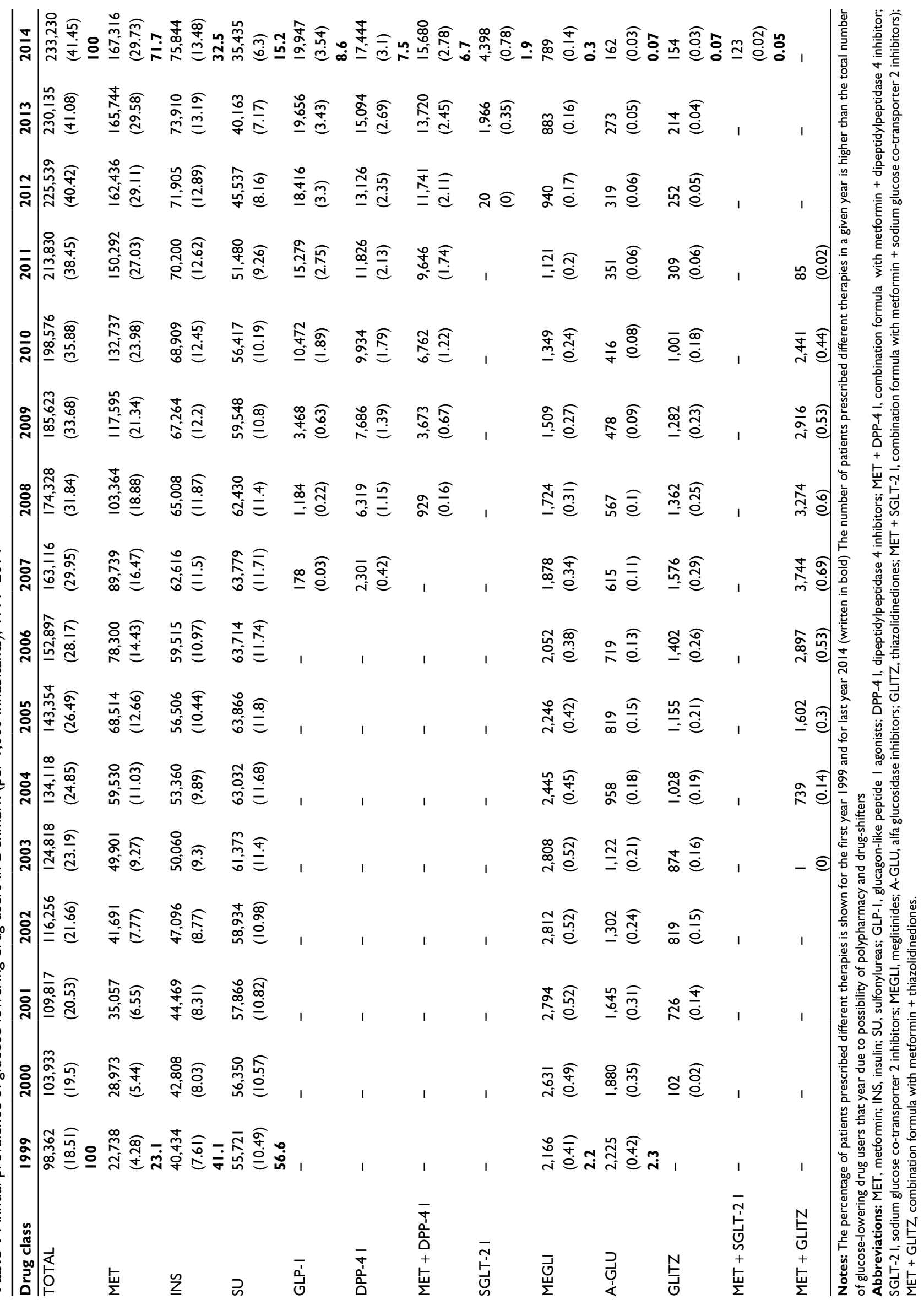




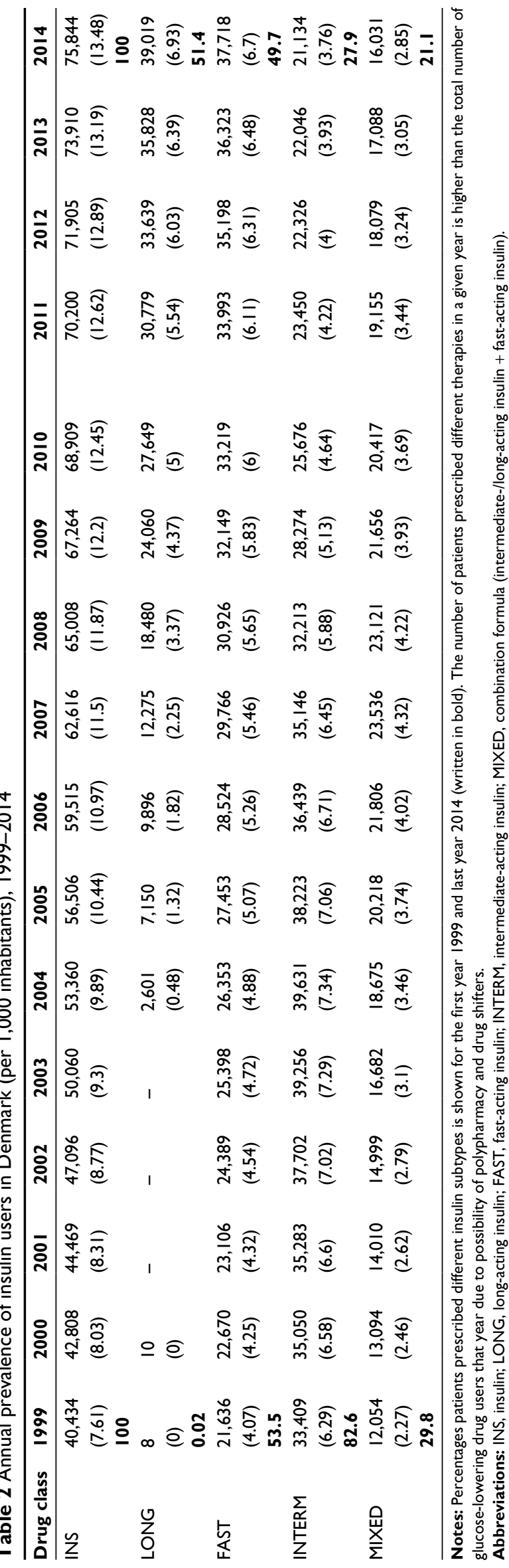

declined from 2004 onward except in the 20- to 39-year-old group (Tables S10-S14 and Figures S3-S7).

\section{Discussion}

From 1999 to 2014, the number of users of any glucose-lowering drug in the Danish population is more than doubled. In accordance with guidelines, in particular the use of metformin has increased, but an increase in users of the recently introduced glucose-lowering drugs was also observed. The number of sulfonylurea and thiazolidinediones users declined much during the second half of the study period. The total number of insulin users increased, primarily caused by long-acting insulin use.

A recent publication based on THIN primary care data from the UK found glucose-lowering prescription patterns 2000-2013 similar to the Danish data, with some important differences. ${ }^{10}$ The use of sulfonylureas decreased to a much greater extent in Denmark (UK: from $64.8 \%$ to $41.4 \%$ and Denmark: from $56.6 \%$ to $15.2 \%$ ). Moreover, the proportion of glucose-lowering drug users who were on insulin was considerably lower and more stable in the UK (20\%-24\% during 2000-2013) than in Denmark (41\%-33\% during 2004-2014). ${ }^{10}$ Annual glucose-lowering drug consumption (measured in DDDs per 1,000 inhabitants per day) in Portugal and the Netherlands appeared to increase with only $32 \%$ and $13 \%$, respectively, between 2004 and $2013,{ }^{11}$ compared with the $\sim 65 \%$ increase in the prevalence of glucose-lowering drug users in Denmark during the same period. Different units of measurement may have contributed to these differences. For example, it is our experience that daily doses of metformin used in clinical practice are often much lower than the metformin DDD (ie, $500 \mathrm{mg}$ or $1,000 \mathrm{mg}$ vs $2,000 \mathrm{mg}$ ). ${ }^{12}$ Increases in DDDs per 1,000 per day may therefore underestimate the true increase in numbers of individual metformin users. Compared to Denmark, the increase in use of DPP-4 inhibitors in Portugal was much more pronounced, whereas data from the Netherland showed a lower use of DPP-4 inhibitors at less than five DDDs per 1,000 per day. Moreover, the decrease of SU use in the Netherland was less pronounced than that in Denmark (DDDs per 1,000 per day $\approx 30$ in 2004, 23 in 2013). ${ }^{11}$ American (2003-2012) ${ }^{13}$ and Japanese (2005-2011) ${ }^{14}$ data also showed an increasing use of metformin and the newer drug classes, whereas the use of sulfonylureas and thiazolidinediones declined. The observed decrease in thiazolidinedione sale from 2007 onward can probably be ascribed to cardiovascular and other safety alerts in the late $2000 \mathrm{~s} .{ }^{15,16}$ The substantially increasing number of users of glucose-lowering drugs in 
general is likely explained by several factors including 1) increased incidence of type $2 \mathrm{DM}$ related to increasing prevalence of obesity and other risk factors (as the observed increasing number of metformin users even among the youngest suggest), 2) increasing prevalence of type 2DM due to younger age at diagnosis and improved therapy and prognosis, ${ }^{17}$ and 3) a more aggressive treatment strategy, with drug treatment of a larger proportion of persons with type $2 \mathrm{DM} .^{12}$

By using Medstat, we were able to obtain complete sales data on glucose-lowering drugs in Denmark during a 16-year period. Limitations of our study include lack of information on whether drugs were prescribed as initiation or add-on therapy and lack of stratification for type 1/type 2DM when assessing insulin use. The GLP-1 receptor agonists, SGLT-2 inhibitors, and the combination pills containing metformin + DPP-4 inhibitor were assessed as single drugs in the Medstat statistics, not as drug classes. Therefore, the number of users of each drug in a subclass was added, and the number of users in a given year might be overestimated to the extent that individuals used more than one drug within a drug class. Finally, complete information on the indication for prescribing a given drug is not currently available, eg, we were not able to distinguish whether metformin was prescribed for diabetes therapy or for treatment of women with polycystic ovarian syndrome in some cases. The latter may account for some of the metformin use observed in the younger age groups. Future more detailed studies based on individual-level longitudinal prescription data linked to registries holding disease diagnoses may overcome some of these limitations and enable studies on polypharmacy, second- and third-line therapies, and glucose-lowering drug adherence and effectiveness. ${ }^{18}$

\section{Conclusion}

This report provides evidence of a more than twofold increase of glucose-lowering drug use during 1999-2014. The pattern of glucose-lowering drug use has changed substantially, reflecting the recommendations of metformin as first-line treatment and the introduction of newer glucose-lowering drug classes that are increasingly used.

\section{Disclosure}

The authors report no conflicts of interest in this work. The Department of Clinical Epidemiology, Aarhus University Hospital, is a member of the Danish Centre for Strategic Research in Type 2 Diabetes (DD2) (Danish Research
Council, grants 09-075724 and 10-079102). DD2 is also supported by the Danish Health and Medicines Authority, the Danish Diabetes Association, and an unrestricted donation from Novo Nordisk A/S. Partners in the DD2 project are listed on the project website at www.DD2.nu. The Department of Clinical Epidemiology, Aarhus University Hospital participates in the International Diabetic Neuropathy Consortium (IDNC) research program, which is supported by a Novo Nordisk Foundation Challenge program grant (grant number NNF14SA0006). The Department of Clinical Epidemiology, Aarhus University Hospital receives funding for other studies from companies in the form of research grants to (and administered by) Aarhus University. None of these studies have any relation to the present study.

\section{References}

1. McGuire H, Longson D, Adler A, Farmer A, Lewin I; Guideline Development Group. Management of type 2 diabetes in adults: summary of updated NICE guidance. BMJ. 2016;353:11575.

2. Inzucchi SE, Bergenstal RM, Buse JB, et al. Management of hyperglycemia in type 2 diabetes, 2015: a patient-centered approach: update to a position statement of the American Diabetes Association and the European Association for the Study of Diabetes. Diabetes Care. 2015;38(1):140-149.

3. International Diabetes Federation Guideline Development Group. Global guideline for type 2 diabetes. Diabetes Res Clin Pract. 2014; 104(1):1-52.

4. Diabetesforeningen [webpage on the Internet]. The National Danish Diabetes Registry; 2016. Available from: http://www.diabetes.dk/presse/ diabetes-i-tal/det-nationale-diabetesregister.aspx. Accessed March 20, 2016.

5. Schmidt M, Pedersen L, Sorensen HT. The Danish Civil Registration System as a tool in epidemiology. Eur J Epidemiol. 2014;29(8):541-549.

6. Danish Serum Institute [webpage on the Internet]. Statistics on annual sales of medicines in Denmark based on the data from the Register of Medicinal Products Statistics; 2016. Available from: www.medstat.dk/en. Accessed January 19, 2016.

7. Kildemoes HW, Sorensen HT, Hallas J. The Danish National Prescription Registry. Scand J Public Health. 2011;39(7 suppl):38-41.

8. Johannesdottir SA, Horvath-Puho E, Ehrenstein V, Schmidt M, Pedersen L, Sorensen HT. Existing data sources for clinical epidemiology: the Danish national database of reimbursed prescriptions. Clin Epidemiol. 2012;4:303-313.

9. Ehrenstein V, Antonsen S, Pedersen L. Existing data sources for clinical epidemiology: Aarhus University Prescription Database. Clin Epidemiol. 2010;2:273-279.

10. Sharma M, Nazareth I, Petersen I. Trends in incidence, prevalence and prescribing in type 2 diabetes mellitus between 2000 and 2013 in primary care: a retrospective cohort study. BMJ Open. 2016;6(1):e010210.

11. Torre C, Guerreiro J, de Oliveira Martins S, Raposo JF, Martins AP, Leufkens H. Patterns of glucose lowering drugs utilization in Portugal and in the Netherlands. Trends over time. Prim Care Diabetes. 2015; 9(6):482-489.

12. Thomsen RW, Baggesen LM, Svensson E, et al. Early glycaemic control among patients with type 2 diabetes and initial glucose-lowering treatment: a 13-year population-based cohort study. Diabetes Obes Metab. 2015;17(8):771-780.

13. Hampp C, Borders-Hemphill V, Moeny DG, Wysowski DK. Use of antidiabetic drugs in the U.S., 2003-2012. Diabetes Care. 2014;37(5): $1367-1374$. 
14. Kohro T, Yamazaki T, Sato H, et al. Trends in antidiabetic prescription patterns in Japan from 2005 to 2011. Int Heart J. 2013;54(2):93-97.

15. European Medicine Agency [webpage on the Internet]. European Medicines Agency recommends new contra-indications and warnings for pioglitazone to reduce small increased risk of bladder cancer. Available from: http://www.ema.europa.eu/ema/index.jsp?curl=pages/ news_and_events/news/2011/07/news_detail_001311.jsp\&mid= WC0b01ac058004d5c1. Accessed January 10, 2016.
16. Ehrenstein V, Hernandez RK, Ulrichsen SP, et al. Rosiglitazone use and post-discontinuation glycaemic control in two European countries, 2000-2010. BMJ Open. 2013;3(9):e003424.

17. Thomsen RW, Sorensen HT. Using registries to identify type 2 diabetes patients. Clin Epidemiol. 2014;7:1-3.

18. Thomsen RW, Baggesen LM, Sogaard M, et al. Effectiveness of intensification therapies in Danes with type 2 diabetes who use basal insulin: a population-based study. Diabet Med. Epub 2016 Jun 9.
Clinical Epidemiology

\section{Publish your work in this journal}

Clinical Epidemiology is an international, peer-reviewed, open access, online journal focusing on disease and drug epidemiology, identification of risk factors and screening procedures to develop optimal preventative initiatives and programs. Specific topics include: diagnosis, prognosis, treatment, screening, prevention, risk factor modification,

Submit your manuscript here: https://www.dovepress.com/clinical-epidemiology-journal

\section{Dovepress}

systematic reviews, risk and safety of medical interventions, epidemiology and biostatistical methods, and evaluation of guidelines, translational medicine, health policies and economic evaluations. The manuscript management system is completely online and includes a very quick and fair peer-review system, which is all easy to use. 